Review Article

\title{
The Effect of Kinesitherapy on Bone Mineral Density in Primary Osteoporosis: A Systematic Review and Meta-Analysis of Randomized Controlled Trial
}

\author{
Shanxi Wang, ${ }^{1}$ Shuzhen Li ${ }^{1},{ }^{1} X$ ing Xie, ${ }^{2}$ and Juying Xie ${ }^{1}$ \\ ${ }^{1}$ College of Rehabilitation, Xiangnan University, Chenzhou 423000, China \\ ${ }^{2}$ Affiliated Hospital of Xiangnan University, Chenzhou 423000, China \\ Correspondence should be addressed to Shuzhen Li; 308634590@qq.com
}

Received 20 May 2020; Accepted 26 June 2020; Published 5 August 2020

Guest Editor: Arham Shabbir

Copyright ( $) 2020$ Shanxi Wang et al. This is an open access article distributed under the Creative Commons Attribution License, which permits unrestricted use, distribution, and reproduction in any medium, provided the original work is properly cited.

Objective. Osteoporosis (OP) is a well-established age-related disease, pathologically characterized by bone microarchitectural deterioration, increased fragility, and low BMD. Primary osteoporosis (POP) is the most common type of OP. Methods. Publications pertaining to the effectiveness of kinesitherapy on BMD in POP from PubMed, SCI, Cochrane Library, Embase, VIP, CNKI, and Wanfang Database were retrieved from their inception to October 2019. Results. A total of 21 studies with 1840 participants were included. The results of the meta-analysis revealed that kinesitherapy plus antiosteoporosis medications had a positive effect on lumbar spine BMD when the duration of intervention was 6 months $\left(\mathrm{MD}=0.11 \mathrm{~g} / \mathrm{cm}^{2} ; 95 \% \mathrm{CI}\right.$ : $0.06-0.15$; $P<0.0001)$ or $>6$ months $\left(\mathrm{MD}=0.04 \mathrm{~g} / \mathrm{cm}^{2} ; 95 \% \mathrm{CI}: 0.02-0.06 ; P<0.0001\right)$ compared with antiosteoporosis medications alone. Additional kinesitherapy plus antiosteoporosis medications were associated with improved femoral neck BMD compared with antiosteoporosis medications alone $\left(\mathrm{MD}=0.09 \mathrm{~g} / \mathrm{cm}^{2} ; 95 \% \mathrm{CI}: 0.03-0.16 ; P=0.004\right)$. Conclusions. Kinesitherapy plus antiosteoporosis medications significantly improved lumbar spine and femoral neck BMD in the current low-quality evidence. Additional high-quality evidence is required to confirm the effect of kinesitherapy on BMD in patients with POP.

\section{Introduction}

With age, bone mass declines, and an accelerated loss of bone mineral density (BMD) occurs by 50 years of age [1]. Osteoporosis (OP) is a well-established age-related disease pathologically characterized by bone microarchitectural deterioration, increased fragility, and low BMD [2]. The diagnosis of OP is based on measurements of BMD and is defined by the World Health Organization as $\mathrm{BMD} \geq 2.5$ standard deviations (SD) below the average value for young healthy individuals [3]. Moreover, OP is associated with high morbidity and mortality, as well as reduced quality of life, which in turn increases the rate of fractures, healthcare costs, and social economic stress [4-9]. In North America [10, 11], over 55 million people are at risk of developing OP or osteopenia. OP is typically classified into three main categories: (1) primary; (2) secondary; and (3) idiopathic.
Primary osteoporosis (POP) refers to the natural aging process of human tissue and organ systems, and the symptoms are associated with degenerative changes in the skeletal system. Moreover, POP is the most common type of OP, accounting for $90 \%$ of OP cases, and includes women with postmenopausal osteoporosis (PMOP) and senile osteoporosis (SOP) $[12,13]$. PMOP is primarily related to postmenopausal estrogen deficiency, whereas SOP is associated with increased age [14]. Thus, as the proportion of elderly populations increases throughout the world, the number of POP cases will also increase gradually. In the European Union, according to relevant statistics [15, 16], 22 million women and 5.5 million men over the age of 50 suffer from osteoporosis. And that number is expected to increase by 23 percent by 2025, according to a study. One study found that the overall prevalence rate of osteoporosis was $32.1 \%$ in at least one measurement site $(28.5 \%$ in the lumbar and 
$14.5 \%$ in the femoral region), while $49.7 \%$ of elderly people suffer from decreased bone mass (osteopenia) in Amirkola, north of Iran [17]. According to the latest epidemiological results of osteoporosis in China, the prevalence of osteoporosis at the age of $40 \sim 49$ is $3.2 \%$, including $2.2 \%$ in males and $4.3 \%$ in females. The prevalence rate of osteoporosis over the age of 50 was $19.2 \%$, including $6.0 \%$ in males and $32.1 \%$ in females. And the prevalence of osteoporosis over the age of 65 was $2.0 \%$, with $0.7 \%$ of men and $51.6 \%$ of women [18].

POP treatment is a long-term process and may not $100 \%$ prevent the development or reverse the symptoms of the disease [19]. In addition, exercise is one of the key recommendations for the prevention and treatment of bone loss [20,21]. Several studies have demonstrated that exercise can prevent bone loss [22] and improve calcium absorption, bone formation [23], and the secretion of sex hormones $[24,25]$, which then promotes BMD [26]. Kinesitherapy, as a part of physical therapy, is a comprehensive exercise that represents one of the most important aspects of medical rehabilitation. Kinesiotherapy involves the movement of various parts of the body or the entire body using exercises to maintain, establish, develop, and change the function of the locomotor apparatus and organs in locomotion. Kinesiotherapy for the treatment of POP primarily includes routine static training, walking training, grip training, outbreak and endurance exercise training, push-ups, stretching, or isometric exercise. In addition, multiple reviews have confirmed that exercise reduces bone loss and increases BMD in postmenopausal women or PMOP [27-29]. A meta-analysis also demonstrated that exercise can improve functional outcomes, including mobility, balance, and self-reported measures of functioning in persons with OP [30]. However, there was no systematic review to evaluate the effect of kinesitherapy on BMD in patients with POP. Therefore, the aim of this study was to conduct a systematic review and meta-analysis to assess the effect of kinesitherapy in persons with POP on lumbar spine and femoral neck BMD via conducting a maximal search of both Chinese and English databases.

\section{Methods}

2.1. Eligibility Criteria. Available human, clinical, or community studies with a randomized controlled trial published in English or Chinese were included in this review. The participants consisted of patients with POP who had no thoracolumbar vertebral fracture and other complications such as heart, vein, lung, liver, and kidney as well as metabolic diseases and were not taking drugs affecting bone metabolism. The age and gender of the subjects were not limited. The included studies focused on the effect of kinesitherapy plus antiosteoporosis medications therapy as a kinesitherapy group compared with antiosteoporosis medications therapy as a control group for the BMD of POP (SOP and SMOP). Those which compared kinesitherapy alone with another exercise or any other antiosteoporosis intervention were excluded. The kinesitherapy should include weight-bearing, impact, resistance, endurance training, or a combination of these types of training, and only single-motion experiments will be ruled out. Health education can be added to all patients, and all inpatients can be given routine care. The outcomes included at least lumbar spine or femoral neck BMD.

2.2. Data Sources and Searches. The original research articles were obtained after a search of six electronic English and Chinese databases, which included PubMed, Science Citation Index (SCI), EMBASE, Chinese Scientific Journal Database (VIP), China National Knowledge Information (CNKI) database, and Wanfang from their inception to October 3, 2019. We used the following search strategy ([kinesitherapy OR exercise] AND osteoporosis AND bone mineral density) in all the English and Chinese databases.

2.3. Study Identification and Quality Assessment. Two reviewers (WSX and LSZ) independently screened and reviewed the title and abstract of the searched studies using NoteExpress V3.2.0.6992 software. The full text of the studies that potentially met the eligibility criteria was obtained, and any potentially relevant references were retrieved according to predefined eligibility criteria. The data were extracted by one reviewer (WSX) using the prepared forms and checked for accuracy by the second reviewer (LSZ). The details extracted from the eligible literature included the language of publication, participant characteristics, sample size, methodological information, participant demographics, experimental and control interventions (category, intensity, frequency, duration, and details of antiosteoporosis medication treatment), outcomes, and adverse effects [31]. Studies published in multiple reports were only included once to avoid duplication in this review. Disagreements were resolved through discussion, and the original author was contacted if an agreement could not be reached. The primary outcomes were lumbar spine BMD and femoral neck BMD, which were expressed as $\mathrm{g} / \mathrm{cm}^{2}$ assessed by dual X-ray absorptiometry or dual photo absorptiometry. The baseline and follow-up data pertaining to BMD were calculated. If follow-up data could not be obtained, the data at the end of the intervention were used instead.

The quality of the studies was independently evaluated by two reviewers (WSX and LSZ) using the Cochrane Collaboration's tool for assessing the risk of bias [32]. The following recommended domains were considered: selection bias (random sequence generation and allocation concealment); performance bias (blinding of participants and personnel); detection bias (blinding of outcome assessment); attrition bias (incomplete outcome data); reporting bias (selective reporting); and other bias, each of which was rated according to the level of bias and categorized as either low, high, or unclear.

2.4. Data Analysis. Review Manager 5.2 software from the Cochrane Collaboration was applied for the data analysis. Statistical heterogeneity among the studies was assessed using a chi-square test or by calculating Higgins $I^{2}$ values 
[33]. The results were pooled using a fixed effect model when the $I^{2}$ value was less than $40 \%$. Otherwise, a random effect model was applied. However, if the $I^{2}$ value among the studies was greater than $75 \%$, the heterogeneity was considered to be substantive and the overall meta-analysis was not appropriate to conduct, but a sensitivity analysis was considered to measure the pooled effect. Subgroup analysis was used to explore the source of heterogeneity if the duration of the intervention exhibited variability. The continuous outcomes were calculated for the mean difference (MD) with standard deviations (SDs) with a corresponding 95\% confidence interval $(\mathrm{CI})$, and all $P$ values were two sided.

\section{Results}

3.1. Description of Studies. A detailed screening flowchart depicting the generation of eligible articles is presented in Figure 1. A total of 791 records were identified via database searches. After removing duplicates, 507 remained to be screened for eligibility. Consequently, 21 studies met the inclusion criteria and were included in the meta-analysis.

Table 1 presents the sample, intervention, and outcome characteristics. This review involved a total of 1840 POP patients (including SOP and PMOP). Part of the subjects were from outpatient clinics, inpatient settings, and community or physical examination centers, with the exception of eleven subjects for whom the sources were unknown. In the included studies, the subject type consisted of eleven PMOP patients, five POP patients, and five SOP patients. In one RCT, the diagnostic criteria for the study were in accordance with the WHO diagnostic criteria for osteoporosis. In the seven RCTs, the reference diagnostic criteria for osteoporosis were Chinese, with one diagnostic criterion for osteoporosis in Japan; the Chinese diagnostic criteria included the Chinese recommended diagnostic criteria for osteoporosis, the Chinese medical association osteoporosis diagnosis and treatment guidelines (a second draft) and a primary bone guide formulated by Chinese Medical Association Osteoporosis and Bone Mineral Disease Branch; one RCT used Western diagnostic criteria for disease; four RCTs only mentioned the diagnostic criteria in accordance with the diagnostic criteria of PMOP or POP; and five RCTs used the laboratory examination in which the $T$ value of was less than or equal to $-2.5 \mathrm{SD}$ in at least one site; the diagnostic criteria in the remaining RCT did not elaborate. The whole RCTs compared kinesitherapy plus medication treatment with medication treatment alone. Kinesitherapy involves comprehensive exercises rather than individual exercises. Of those RCTs, only one RCT used traditional Chinese medicine (e.g., kidney method), and the remaining RCTs were treated with Western medicine. All of the RCTs included a measure of lumbar spine BMD, and five of the RCTs included lumbar spine and femoral neck BMD.

3.2. Methodological Quality. As shown in Figure 2, twelve studies described the generation of random sequences. Five of these studies used a random number table, three of these used simple random methods, three studies used computergenerated random numbers, and the remaining one trial used the method of lottery. Three trials involve allocation concealment. However, the blind intervention associated with the intervention exercises cannot be implemented blindly. One study described that the data analysis was based on the author's own statistics. One study described an exit from the case, but did not explain the reason. There were no dropouts indicated or explanations for withdrawal in the remaining studies. All of the included studies were considered to have a high risk of bias.

3.3. Meta-Analysis of Lumbar Spine BMD. All the controls in the literatures were kinesitherapy plus antiosteoporosis drugs versus antiosteoporosis drugs. According to the duration of the intervention, the subgroups were divided into three groups based on an intervention duration of (1) less than 6 months; (2) 6 months; and (3) longer than 6 months.

3.3.1. Intervention Duration < 6 Months. Two trials $[35,53]$ compared the effect of kinesitherapy plus antiosteoporosis medications with antiosteoporosis medications alone on lumbar spine BMD when the duration of intervention was less than 6 months. The meta-analyses indicated that there was no significant difference between the two groups $(\mathrm{MD}=0.02 ; 95 \% \mathrm{CI}:-0.00-0.05 ; P=0.10)$ (Figure 3 ).

3.3.2. Intervention Duration $=6$ Months. Ten studies $[37,38,41,46,51,54]$ involving 699 participants reported that after 6 months, kinesitherapy had significantly increased lumbar $\mathrm{BMD} \quad\left(\mathrm{MD}=0.11 \mathrm{~g} / \mathrm{cm}^{2} ; \quad 95 \% \quad \mathrm{CI}: \quad 0.06-0.15\right.$; $P<0.0001)$. However, the heterogeneity among the studies was substantial with $I^{2}=83 \%$, and no obvious changes were observed after the sensitivity analysis when any one or two of the studies were removed (Figure 4).

3.3.3. Intervention Duration $>6$ Months. Eleven studies $[34,36,39,40,42-44,48-50,52]$ involving 1019 participants revealed that when the duration of treatment was longer than 6 months, the lumbar spine BMD in the kinesitherapy group significantly increased compared with the control group $\left(\mathrm{MD}=0.04 \mathrm{~g} / \mathrm{cm}^{2} ; 95 \% \mathrm{CI}: 0.02-0.06 ; P<0.0001\right)$ with high heterogeneity $\left(I^{2}=73 \%\right)$ (Figure 5$)$. The heterogeneity was $I^{2}=23 \%$ after the sensitivity analysis when one study [40] was removed.

3.4. Meta-Analysis of Femoral Neck BMD. Five trials [43-45, 47, 53] involving 439 participants compared effect of kinesitherapy plus antiosteoporosis medications with antiosteoporosis medications alone on lumbar spine BMD. The meta-analysis revealed a significant antiosteoporosis effect on lumbar spine BMD (MD =0.09 g/ $\mathrm{cm}^{2} ; 95 \% \mathrm{CI}$ : 0.03-0.16; $P=0.004)$ but with high heterogeneity $\left(I^{2}=90 \%\right)$ (Figure 6). It showed low heterogeneity $\left(I^{2}=0 \%\right)$ after the sensitivity analysis when two studies were removed $[45,47]$. 


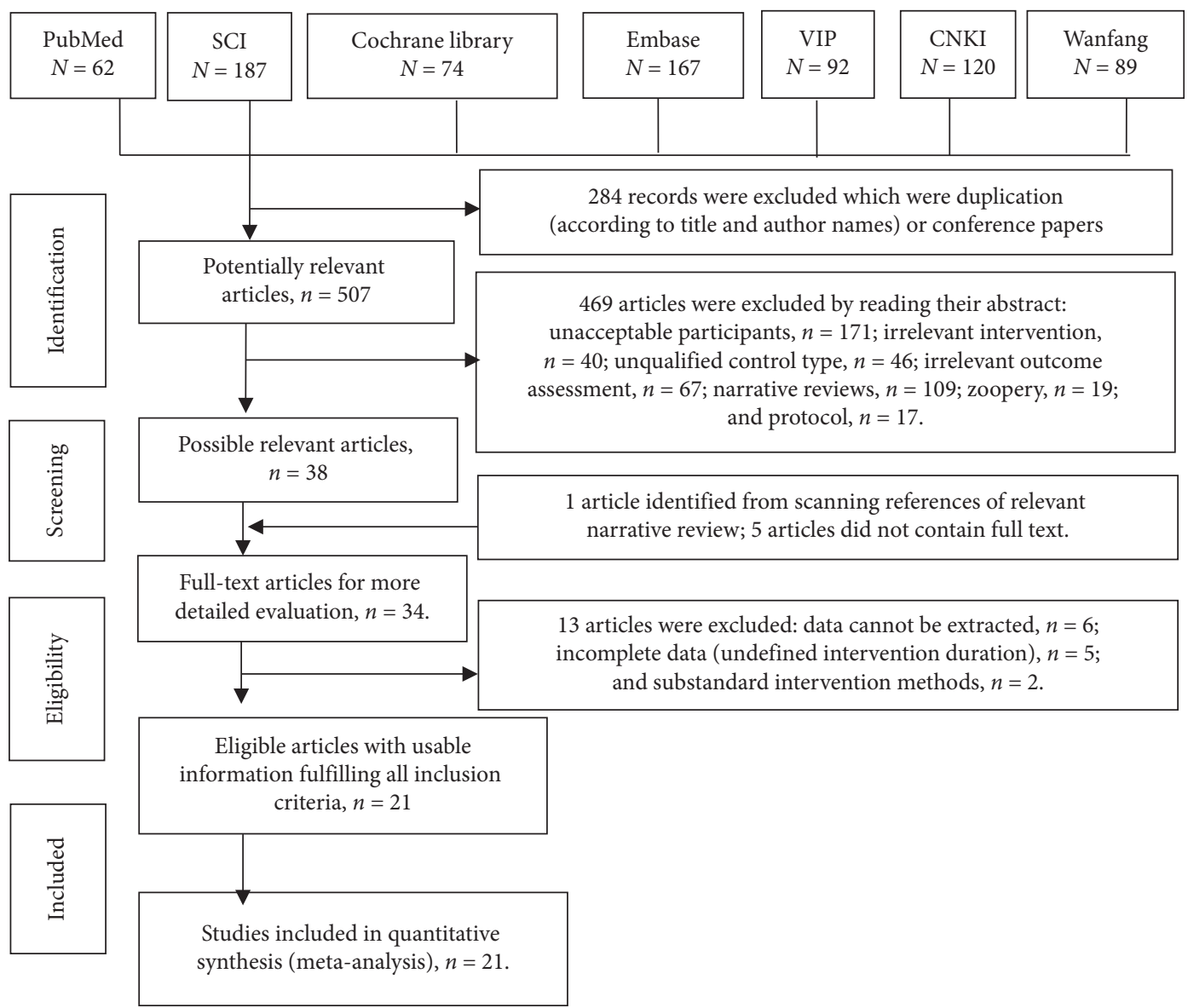

FIgURE 1: Details of literature search and selection. SCI: Science Citation Index; VIP: Chinese Scientific Journal Database; CNKI: China National Knowledge Information database.

TABLE 1: The characteristics of all the trails.

\begin{tabular}{|c|c|c|c|c|c|}
\hline \multirow{2}{*}{ Author, year } & \multirow{2}{*}{$\begin{array}{l}\text { Participants (type, } \\
\text { source, age, sample) }\end{array}$} & \multirow{2}{*}{$\begin{array}{l}\text { Duration } \\
\text { (months) }\end{array}$} & \multicolumn{2}{|c|}{ Intervention } & \multirow{2}{*}{ Outcomes } \\
\hline & & & Kinesitherapy group & Control group & \\
\hline $\begin{array}{l}\text { Iwamoto } \\
\text { et al., } 2001 \\
{[34]}\end{array}$ & $\begin{array}{l}\text { PMOP, unspecified, } \\
\text { 53-77 years, } 28 \text { (KT: } \\
\text { 8, CON: 20) }\end{array}$ & 24 & $\begin{array}{l}\text { Brisk walking (1000 steps in the first } \\
7 \text { days, increase the step count by } \\
30 \% / \text { week) }+ \text { gymnastic training } \\
\text { (no details provided) }+ \text { CON }\end{array}$ & $\begin{array}{l}\text { Calcium lactate }(2.0 \mathrm{~g}, \mathrm{Qd}) \text { and } \\
1 \alpha \text {-hydroxyvitamin D3 }(1 \mu \mathrm{g}, \mathrm{Qd})\end{array}$ & $\begin{array}{l}\text { Lumbar spine } \\
\text { BMD }\end{array}$ \\
\hline $\begin{array}{l}\text { Shen, } 2003 \\
\text { [35] }\end{array}$ & $\begin{array}{l}\text { POP, outpatient and } \\
\text { inpatient, } 45-80 \\
\text { years, } 60 \text { (KT: } 30 \text {, } \\
\text { CON: } 30) \\
\end{array}$ & 3 & $\begin{array}{l}\text { Aerobics, tai chi, dance, yangko, } \\
\text { jogging, walking etc. }(30-60 \mathrm{~min} / \\
\text { time, } 5-7 \text { times/week) }+\mathrm{CON}\end{array}$ & $\begin{array}{l}\text { Tonifying kidney granules ( } 3 \\
\text { times/day, } 1 \text { dose/time) }\end{array}$ & $\begin{array}{l}\text { Lumbar spine } \\
\text { BMD }\end{array}$ \\
\hline $\begin{array}{l}\text { Zhu, } 2007 \\
{[36]}\end{array}$ & $\begin{array}{l}\text { SOP, outpatient, } \\
\text { 60-72 years, } 96(\mathrm{KT}: \\
\text { 48, CON: } 48)\end{array}$ & 12 & $\begin{array}{l}\text { Walking, jogging, tai chi } \\
\text { (30-60 min/time, 3-5 times/ } \\
\text { week })+ \text { CON }\end{array}$ & Calcium $(600 \mathrm{mg} / \mathrm{d})$ & $\begin{array}{l}\text { Lumbar spine } \\
\text { BMD }\end{array}$ \\
\hline $\begin{array}{l}\text { Liu et al., } \\
2007 \text { [37] }\end{array}$ & $\begin{array}{l}\text { PMOP, outpatient, } \\
\text { 48-61 years, } 68 \text { (KT: } \\
\text { 36, CON: } 32)\end{array}$ & 6 & $\begin{array}{c}\text { Draft movement, abdominal } \\
\text { isometric exercises, flexion, and } \\
\text { extension of the upper limbs ( } 20 \\
\text { minutes each time, once every } 3 \\
\text { days) + CON }\end{array}$ & Caltrate D (600 mg, Qd) & $\begin{array}{l}\text { Lumbar spine } \\
\text { BMD }\end{array}$ \\
\hline $\begin{array}{l}\text { Li et al., } 2008 \\
\text { [38] }\end{array}$ & $\begin{array}{l}\text { PMOP, unspecified, } \\
\text { 48-61 years, } 70 \text { (KT: } \\
\text { 38, CON: } 32)\end{array}$ & 6 & $\begin{array}{c}\text { Draft movement, abdominal } \\
\text { isometric exercises, flexion, and } \\
\text { extension of the upper limbs } \\
(20 \text { min/time, once every four } \\
\text { days })+ \text { CON }\end{array}$ & $\begin{array}{l}\text { Ossotide injection }(50 \mathrm{mg}, \mathrm{Qd} \\
20 \text { days in total) }\end{array}$ & $\begin{array}{c}\text { Lumbar spine } \\
\text { BMD }\end{array}$ \\
\hline $\begin{array}{l}\text { Liu et al., } \\
2009[39]\end{array}$ & $\begin{array}{c}\text { SOP, outpatient } \\
\text { service, } 60-94 \text { years, } \\
60 \text { (KT: } 30, \text { CON: } \\
30)\end{array}$ & 12 & $\begin{array}{l}\text { Walking, jogging, tai chi }(60 \mathrm{~min} / \\
\text { time, } 1 \text { time/day })+\mathrm{CON}\end{array}$ & $\begin{array}{l}\text { Fosamax (10 mg, once a day }) \text { and } \\
\text { calcium }(600 \mathrm{mg} / \mathrm{d})\end{array}$ & $\begin{array}{l}\text { Lumbar spine } \\
\text { BMD }\end{array}$ \\
\hline
\end{tabular}


TABLE 1: Continued.

\begin{tabular}{|c|c|c|c|c|c|}
\hline \multirow{2}{*}{ Author, year } & \multirow{2}{*}{$\begin{array}{c}\text { Participants (type, } \\
\text { source, age, sample) }\end{array}$} & \multirow{2}{*}{$\begin{array}{l}\text { Duration } \\
\text { (months) }\end{array}$} & \multicolumn{2}{|c|}{ Intervention } & \multirow{2}{*}{ Outcomes } \\
\hline & & & Kinesitherapy group & Control group & \\
\hline $\begin{array}{l}\text { Liu and } \\
\text { Wang, } 2012 \\
{[40]}\end{array}$ & $\begin{array}{c}\text { SOP, unspecified, } \\
60-81 \text { years, } 320 \\
\text { (KT: } 162, \text { CON: } 158) \\
\end{array}$ & 12 & $\begin{array}{l}\text { Tai chi and jogging (no less than } \\
30 \mathrm{~min} / \text { time, no less than } 4 \text { times/ } \\
\text { week) + CON }\end{array}$ & $\begin{array}{c}\text { Calcium carbonate } \mathrm{D}_{3}(600 \mathrm{mg} \text {, } \\
\text { Qd) }\end{array}$ & $\begin{array}{l}\text { Lumbar spine } \\
\text { BMD }\end{array}$ \\
\hline $\begin{array}{l}\text { Li et al., } 2013 \\
\text { [41] }\end{array}$ & $\begin{array}{l}\text { SOP, hospital, } 67 \pm 4 \\
\text { years, } 86(\mathrm{KT}: 43 \\
\text { CON: } 43)\end{array}$ & 6 & $\begin{array}{l}\text { Progressive lumbar dorsal muscle } \\
\text { function exercise includes sitting } \\
\text { training, swallow training and five- } \\
\text { point support training (1-2 times/ } \\
\text { day) + CON }\end{array}$ & $\begin{array}{l}\text { Lorelli calcium capsule ( } 1 \\
\text { capsules, } 1 \text { time/d for } 2 \\
\text { consecutive months) }\end{array}$ & $\begin{array}{l}\text { Lumbar spine } \\
\text { BMD }\end{array}$ \\
\hline $\begin{array}{l}\text { Ming, } 2013 \\
{[42]}\end{array}$ & $\begin{array}{l}\text { SOP, hospital, 60-78 } \\
\text { years, } 96 \text { (KT: } 48, \\
\text { CON: } 48) \\
\end{array}$ & 12 & $\begin{array}{l}\text { Walking, aerobics, running ,and } \\
\text { tai chi ( } 5 \text { to } 7 \text { times a week for } 45 \text { to } \\
60 \text { minutes })+ \text { CON }\end{array}$ & $\begin{array}{c}\text { Calcium gluconate }(20 \mathrm{ml} / \text { time, } 3 \\
\text { times/day) and vitamin } \mathrm{D}(400 \\
\text { units, } 2 \text { times/day) }\end{array}$ & $\begin{array}{l}\text { Lumbar spine } \\
\text { BMD }\end{array}$ \\
\hline $\begin{array}{l}\text { Chen, } 2015 \\
{[43]}\end{array}$ & $\begin{array}{l}\text { PMOP, clinic, } 53- \\
\text { years, } 57 \text { (KT: } 27 \\
\quad \text { CON: } 30)\end{array}$ & 12 & $\begin{array}{l}\text { Brisk walking (15-30 minutes), } \\
\text { resistance strength exercises } \\
\text { (15-20 minutes), and balance and } \\
\text { flexibility exercises ( } \text { simplify tai chi } \\
\text { and five birds, } 15-20 \mathrm{~min})+\mathrm{CON}\end{array}$ & $\begin{array}{l}\text { Alendronate }(70 \mathrm{mg}, \mathrm{Qd}) \text {, caltrate } \\
\mathrm{D}(600 \mathrm{mg}, \mathrm{Qd}) \text {, and alfacalcidol } \\
\text { soft capsules }(0.25 \mu \mathrm{g}, \mathrm{Qd})\end{array}$ & $\begin{array}{l}\text { Lumbar spine } \\
\text { and femoral } \\
\text { neck BMD }\end{array}$ \\
\hline $\begin{array}{l}\text { Dischereit } \\
\text { et al., } 2016 \\
{[44]}\end{array}$ & $\begin{array}{l}\text { PMOP, unspecified, } \\
68 \text { years, } 42(\mathrm{KT}: 25, \\
\text { CON: } 17)\end{array}$ & 24 & $\begin{array}{l}\text { Endurance and strength training } \\
\text { program (3 sessions once weekly, } \\
65 \mathrm{~min})+\mathrm{CON}\end{array}$ & $\begin{array}{c}\text { Adequate calcium and vitamin D } \\
\text { supplementation and } \\
\text { bisphosphonate therapy }\end{array}$ & $\begin{array}{c}\text { Lumbar spine } \\
\text { and femoral } \\
\text { neck BMD }\end{array}$ \\
\hline $\begin{array}{l}\text { Li et al., } 2016 \\
{[45]}\end{array}$ & $\begin{array}{l}\text { PMOP, unspecified, } \\
\text { 52-76 years, } 188 \\
\text { (KT: 94, CON: } 94)\end{array}$ & 6 & $\begin{array}{l}\text { Mainly includes walking, aerobics, } \\
\text { running, and tai chi }(30-60 \mathrm{~min} / \\
\text { time, more than } 3 \text { times/ } \\
\text { week })+ \text { CON }\end{array}$ & $\begin{array}{l}\text { Caltrate } \mathrm{D}(1000 \mathrm{mg}, \mathrm{Qd}) \text {, } \\
\text { derivatives, vitamin } \mathrm{D} \text {, and } \\
\text { raloxifene (1 pill, Qd) }\end{array}$ & $\begin{array}{l}\text { Lumbar spine } \\
\text { and femoral } \\
\text { neck BMD }\end{array}$ \\
\hline $\begin{array}{l}\text { Chen, } 2016 \\
{[46]}\end{array}$ & $\begin{array}{l}\text { PMOP, unspecified, } \\
\text { 53-77 years, 65(KT: } \\
\text { 33, CON:32) }\end{array}$ & 6 & $\begin{array}{l}\text { Brisk walking and tai chi } \\
(30-50 \text { min/time, } 2-3 \text { times/ } \\
\text { week })+ \text { CON }\end{array}$ & $\begin{array}{l}\text { Alendronate }(70 \mathrm{mg}, \mathrm{Qd}) \text {, caltrate } \\
\mathrm{D}(600 \mathrm{mg}, \mathrm{Qd}) \text {, and alfacalcidol } \\
\text { soft capsules }(0.25 \mu \mathrm{g}, \mathrm{Qd})\end{array}$ & $\begin{array}{l}\text { Lumbar spine } \\
\text { BMD }\end{array}$ \\
\hline $\begin{array}{l}\mathrm{Xu}, 2017 \\
{[47]}\end{array}$ & $\begin{array}{l}\text { PMOP, unspecified, } \\
\text { 51-67 years, } 100 \\
\text { (KT:50, CON:50) } \\
\end{array}$ & 6 & $\begin{array}{c}\text { Aerobics, tai chi, and jogging } \\
\text { (more than } 30 \mathrm{~min} \text {, more than } 3 \\
\text { times/week) }+ \text { CON }\end{array}$ & $\begin{array}{c}\text { Calcine D (2 times/day, 2pills/ } \\
\text { time })+ \text { estrogen ( } 1 \text { time/day, } \\
60 \mathrm{mg} / \text { time })\end{array}$ & $\begin{array}{c}\text { Lumbar spine } \\
\text { and femoral } \\
\text { neck BMD }\end{array}$ \\
\hline $\begin{array}{l}\text { Chang, } 2017 \\
{[48]}\end{array}$ & $\begin{array}{l}\text { POP, unspecified, } \\
\text { 60-79 years, } 84 \text { (KT: } \\
\text { 42, CON: } 42)\end{array}$ & 12 & $\begin{array}{l}\text { Aerobics, walking, swimming, } \\
\text { jogging, and cycling ( } 3-4 \text { times, } \\
\text { not less than } 2 \text { times, each exercise } \\
30-60 \text { minutes) + CON }\end{array}$ & $\begin{array}{l}\text { Calcine D } 600 \text { ( } 1 \text { tablet once, } 2 \\
\text { times a day) and health education }\end{array}$ & $\begin{array}{l}\text { Lumbar spine } \\
\text { BMD }\end{array}$ \\
\hline Qi, 2017 [49] & $\begin{array}{l}\text { PMOP, community } \\
\text { healthcare center, } \\
45-65 \text { years, } 56 \text { (KT: } \\
\text { 28, CON:28) }\end{array}$ & 12 & $\begin{array}{l}\text { Aerobic exercise resistance group, } \\
\text { load bearing, and stretching } \\
(30 \mathrm{~min} / \text { time, } 3-5 \text { times/week, } \\
\text { more than } 1 \mathrm{~h})+\mathrm{CON}\end{array}$ & Conventional treatment & $\begin{array}{l}\text { Lumbar spine } \\
\text { BMD }\end{array}$ \\
\hline $\begin{array}{l}\text { Wen, } 2017 \\
{[50]}\end{array}$ & $\begin{array}{l}\text { POP, unspecified, } \\
\text { 60-78 years, } 96 \text { (KT: } \\
\text { 48, CON: } 48)\end{array}$ & 12 & Jogging, tai chi, etc. (daily) + CON & $\begin{array}{l}\text { Routine prevention and taking } \\
\text { medicine }\end{array}$ & $\begin{array}{l}\text { Lumbar spine } \\
\text { BMD }\end{array}$ \\
\hline $\begin{array}{l}\text { Liu and } \\
\text { Yang, } 2018 \\
{[51]}\end{array}$ & $\begin{array}{l}\text { POP, unspecified, } \\
\text { 36-79 years, } 80 \text { (KT: } \\
\text { 40, CON: } 40)\end{array}$ & 6 & $\begin{array}{l}\text { Walking, fitness running, } \\
\text { ballroom dancing, and swimming } \\
\text { (at least } 12 \text { times a month, each } \\
\text { time } \geq 30 \mathrm{~min} \text { ) + CON }\end{array}$ & Calcium and vitamin D3 & $\begin{array}{l}\text { Lumbar spine } \\
\text { BMD }\end{array}$ \\
\hline $\begin{array}{l}\text { Zheng et al., } \\
2019 \text { [52] }\end{array}$ & $\begin{array}{l}\text { POP, unspecified, } \\
\text { 53-77 years, } 84 \text { (KT: } \\
\text { 42, CON: } 42)\end{array}$ & 12 & $\begin{array}{l}\text { Walking, jogging, alternate } \\
\text { running, cycling, etc. ( } 3 \text { to } 4 \text { times } \\
\text { per week, the minimum } 2 \text { times, } \\
30-60 \mathrm{~min})+ \text { CON }\end{array}$ & $\begin{array}{c}\text { Calcium (300 mg/tablet, } 2 \text { times/ } \\
\text { d,1 tablet/time })\end{array}$ & $\begin{array}{l}\text { Lumbar spine } \\
\text { BMD }\end{array}$ \\
\hline $\begin{array}{l}\text { Li et al., } 2019 \\
\text { [53] }\end{array}$ & $\begin{array}{l}\text { PMOP, unspecified, } \\
\text { 50-65 years, } 52(\mathrm{KT} \text { : } \\
\text { 26, CON: } 26)\end{array}$ & 3 & $\begin{array}{l}\text { Brisk walking ( } 30 \text { min, once a day, } \\
4 \mathrm{~d} / \text { week) and resistance training } \\
\text { (week } 1,2 \text { weekly complete } 1 \text { set } \\
\text { (15 times/set), and then add } 1 \text { set } \\
\text { every } 2 \text { weeks) + CON }\end{array}$ & $\begin{array}{c}\text { Calcium carbonate D3 }(600 \mathrm{mg}, 1 \\
\text { time/d), alfacalcidol soft capsule } \\
(0.5 \mathrm{~g}, 1 \text { time } / \mathrm{d}) \text {, and sodium } \\
\text { alendronate }(70 \mathrm{mg}, 1 \text { time } / \mathrm{d} / \\
\text { weeks) for } 3 \text { months }\end{array}$ & $\begin{array}{l}\text { Lumbar spine } \\
\text { and femoral } \\
\text { neck BMD }\end{array}$ \\
\hline $\begin{array}{l}\text { Yan et al., } \\
2019 \text { [54] }\end{array}$ & $\begin{array}{l}\text { POP, unspecified, } \\
\text { 53-77 years, } 52(\mathrm{KT}: \\
\text { 26, CON: } 26)\end{array}$ & 6 & $\begin{array}{l}\text { Flexible resistance exercise therapy } \\
\text { to exercise the lumbar and dorsal } \\
\text { muscles ( } 5 \text { times/week) + CON }\end{array}$ & $\begin{array}{l}\text { Calcium carbonate } \mathrm{D} 3(600 \mathrm{mg}) \text {, } \\
\text { vitamin D3 ( } 0.25 \mathrm{UG}) \text {, health } \\
\text { education, and routine nursing }\end{array}$ & $\begin{array}{l}\text { Lumbar spine } \\
\text { BMD }\end{array}$ \\
\hline
\end{tabular}




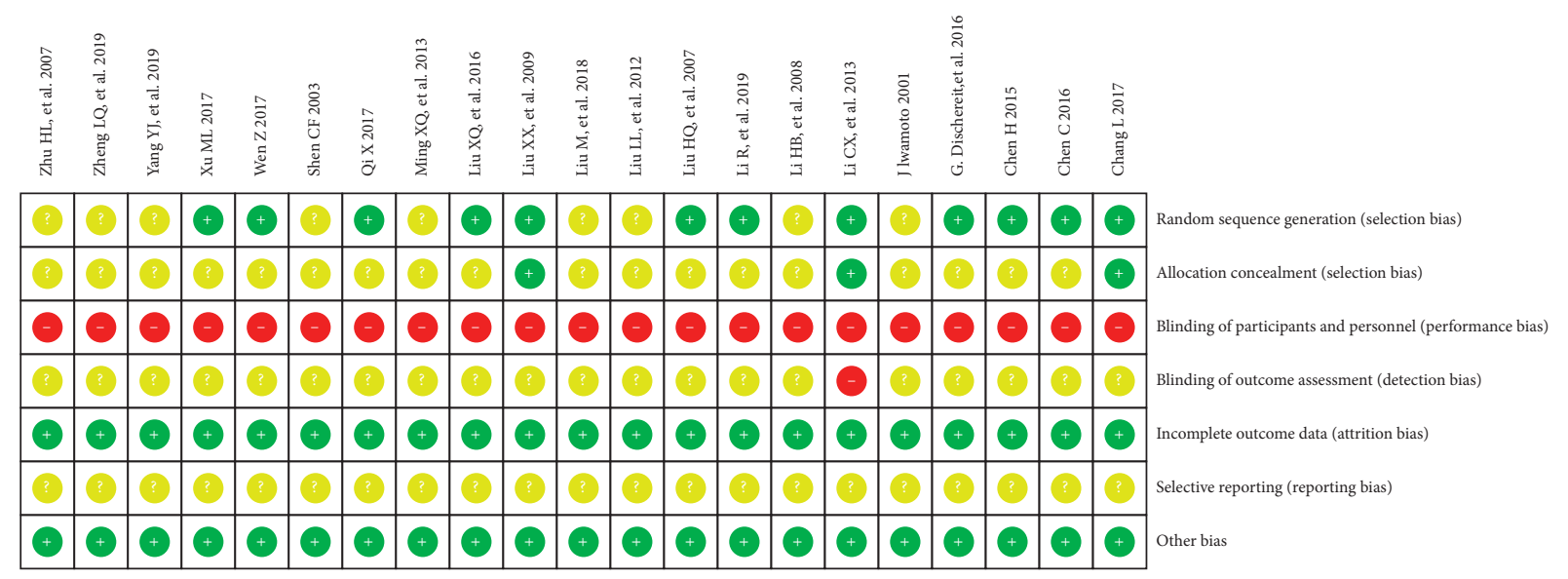

FIGURE 2: Risk of bias summary for each included study.

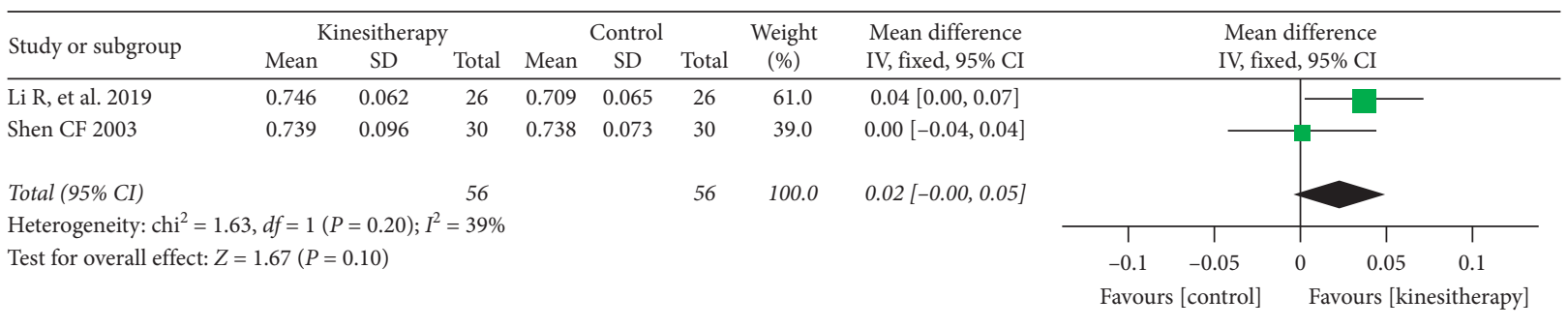

FIGURE 3: Kinesitherapy plus antiosteoporosis medications versus antiosteoporosis medications on lumbar spine BMD (intervention duration $<6$ months).

\begin{tabular}{|c|c|c|c|c|c|c|c|c|c|c|c|c|}
\hline \multirow{3}{*}{$\begin{array}{l}\text { Study or subgroup } \\
\text { Chen C, } 2016\end{array}$} & \multicolumn{3}{|c|}{ Kinesitherapy } & \multicolumn{3}{|c|}{ Control } & \multirow{2}{*}{$\begin{array}{c}\text { Weight } \\
(\%)\end{array}$} & \multirow{2}{*}{$\begin{array}{c}\text { Mean difference } \\
\mathrm{IV} \text {, random, 95\% CI }\end{array}$} & \multirow{2}{*}{\multicolumn{4}{|c|}{$\begin{array}{c}\text { Mean difference } \\
\text { IV, random, } 95 \% \text { CI }\end{array}$}} \\
\hline & Mean & $\mathrm{SD}$ & Total & Mean & $\mathrm{SD}$ & Total & & & & & & \\
\hline & 0.802 & 0.081 & 33 & 0.766 & 0.088 & 32 & 14.1 & $0.04[-0.01,0.08]$ & & & $\square$ & \\
\hline Li CX, et al. 2013 & 1.012 & 0.114 & 43 & 0.855 & 0.103 & 43 & 13.7 & $0.16[0.11,0.20]$ & & & & \\
\hline Li HB, et al. 2008 & 0.53 & 0.19 & 38 & 0.48 & 0.22 & 32 & 9.1 & $0.05[-0.05,0.15]$ & & & & \\
\hline Li XQ, et al. 2016 & 1.02 & 0.1 & 94 & 0.84 & 0.16 & 94 & 14.3 & $0.18[0.14,0.22]$ & & & & - \\
\hline Liu HQ, et al. 2007 & 0.52 & 0.17 & 36 & 0.45 & 0.22 & 32 & 9.3 & $0.07[-0.02,0.16]$ & & & & \\
\hline Liu M, et al. 2018 & 0.83 & 0.11 & 40 & 0.75 & 0.09 & 40 & 13.8 & $0.08[0.04,0.12]$ & & & & \\
\hline XU ML, 2017 & 1.04 & 0.18 & 50 & 0.86 & 0.15 & 50 & 11.9 & $0.18[0.12,0.24]$ & & & & \\
\hline Yan YJ, et al. 2019 & 0.841 & 0.075 & 21 & 0.771 & 0.069 & 21 & 13.9 & $0.07[0.03,0.11]$ & & & - & \\
\hline Total $(95 \%$ CI) & & & 355 & & & 344 & 100.0 & $0.11[0.06,0.15]$ & & & & \\
\hline \multicolumn{9}{|c|}{ Heterogeneity: $\operatorname{tau}^{2}=0.00 ; \mathrm{chi}^{2}=41.00, d f=7(P<0.00001) ; I^{2}=83 \%$} & 1 & 1 & r & 1 \\
\hline \multicolumn{9}{|c|}{ Test for overall effect: $Z=4.64(P<0.00001)$} & -0.2 & -0.1 & 0.1 & 0.2 \\
\hline & & & & & & & & & Favours & control] & Favours [] & nesith \\
\hline
\end{tabular}

FIGURE 4: Kinesitherapy plus antiosteoporosis medications versus antiosteoporosis medications on lumbar spine BMD (intervention duration $=6$ months).

\section{Discussion}

4.1. Summary. POP is a worldwide health problem that primarily impacts postmenopausal women and senile individuals. Moreover, POP is often related to physical frailty, an increased risk of falls, substantial morbidity, mortality, and impairment in quality of life [55]. The aim of OP treatment is to improve BMD and prevent fractures. Nonpharmacological treatment includes a healthy diet, prevention of falls, and physical exercise programs. Pharmacological treatment involves calcium, vitamin $\mathrm{D}$, and medications for activating bone tissue (e.g., antiresorptives, bone formers, and mixed agents) [56]. In addition, exercise is considered important for maintaining bone health. Individuals with OP are strongly recommended to regularly engage in multicomponent exercise programs [57]. Moreover, several studies have confirmed that exercise can increase BMD at the femoral neck and the lumbar spine in elderly women with osteoporosis $[58,59]$. This review is the first systematic review and meta-analysis to evaluate the effect of kinesitherapy on BMD on the lumbar spine and femoral neck in persons with POP from RCTs. This study 


\begin{tabular}{|c|c|c|c|c|c|c|c|c|c|c|c|c|}
\hline \multirow{3}{*}{$\begin{array}{l}\text { Study or subgroup } \\
\text { Chang L, } 2017\end{array}$} & \multicolumn{3}{|c|}{ Kinesitherapy } & \multicolumn{3}{|c|}{ Control } & \multirow{3}{*}{$\begin{array}{c}\begin{array}{c}\text { Weight } \\
(\%)\end{array} \\
5.8\end{array}$} & \multirow{3}{*}{$\begin{array}{c}\text { Mean difference } \\
\text { IV, random, 95\% CI } \\
0.03[-0.04,0.10]\end{array}$} & \multirow{2}{*}{\multicolumn{4}{|c|}{$\begin{array}{c}\text { Mean difference } \\
\text { IV, random, 95\% CI }\end{array}$}} \\
\hline & \multirow{2}{*}{$\begin{array}{l}\text { Mean } \\
0.863\end{array}$} & \multirow{2}{*}{$\frac{\mathrm{SD}}{0.169}$} & \multirow{2}{*}{$\begin{array}{c}\text { Total } \\
42\end{array}$} & \multirow{2}{*}{$\begin{array}{l}\text { Mean } \\
0.834\end{array}$} & \multirow{2}{*}{$\frac{\mathrm{SD}}{0.155}$} & \multirow{2}{*}{$\frac{\text { Total }}{42}$} & & & & & & \\
\hline & & & & & & & & & & & & \\
\hline Chen H, 2015 & 0.795 & 0.085 & 27 & 0.746 & 0.081 & 30 & 10.3 & $0.05[0.01,0.09]$ & & & & \\
\hline G. Dischereit, et al. 2016 & 0.85 & 0.11 & 25 & 0.83 & 0.12 & 17 & 5.6 & $0.02[-0.05,0.09]$ & & & & \\
\hline J lwamoto, 2001 & 0.62 & 0.087 & 8 & 0.616 & 0.044 & 20 & 6.6 & $0.00[-0.06,0.07]$ & & & & \\
\hline Liu LL, et al. 2012 & 0.877 & 0.043 & 162 & 0.865 & 0.049 & 158 & 18.8 & $0.01[0.00,0.02]$ & & & - & \\
\hline Liu XX, et al. 2009 & 0.86 & 0.15 & 30 & 0.83 & 0.13 & 30 & 5.6 & $0.03[-0.04,0.10]$ & & & & \\
\hline Ming XQ, et al. 2013 & 0.904 & 0.16 & 48 & 0.841 & 0.12 & 48 & 7.6 & $0.06[0.01,0.12]$ & & & & \\
\hline Qi X, 2017 & 0.875 & 0.022 & 28 & 0.829 & 0.015 & 28 & 18.9 & $0.05[0.04,0.06]$ & & & - & \\
\hline Wen Z, 2017 & 0.84 & 0.19 & 48 & 0.82 & 0.17 & 48 & 5.5 & $0.02[-0.05,0.09]$ & & & & \\
\hline Zheng LQ, et al. 2019 & 0.749 & 0.151 & 42 & 0.613 & 0.145 & 42 & 6.6 & $0.14[0.07,0.20]$ & & & & \\
\hline Zhu HL, et al. 2007 & 0.901 & 0.15 & 48 & 0.837 & 0.1 & 48 & 8.6 & $0.06[0.01,0.11]$ & & & & \\
\hline Total (95\% CI) & & & 508 & & & 511 & 100.0 & $0.04[0.02,0.06]$ & & & & \\
\hline Heterogeneity: $\operatorname{tau}^{2}=0.00$ & $\operatorname{chi}^{2}=3$ & $.37, d f=$ & $0(P<C$ & $.0001)$ & $2^{2}=73 \%$ & & & & $T$ & & 1 & $T$ \\
\hline Test for overall effect: $Z=$ & $4.05(P<$ & $.0001)$ & & & & & & & -0.2 & -0.1 & 0.1 & 0.2 \\
\hline & & & & & & & & & & $\mathrm{rs}[\mathrm{col}$ & Favours [kin & arapy] \\
\hline
\end{tabular}

FIGURE 5: Kinesitherapy plus antiosteoporosis medications versus antiosteoporosis medications on lumbar spine BMD (intervention duration $>6$ months).

\begin{tabular}{|c|c|c|c|c|c|c|c|c|c|c|c|c|}
\hline \multirow{3}{*}{$\frac{\text { Study or subgroup }}{\text { Chen H, } 2015}$} & \multicolumn{3}{|c|}{ Kinesitherapy } & \multicolumn{3}{|c|}{ Control } & \multirow{2}{*}{$\begin{array}{c}\text { Weight } \\
(\%)\end{array}$} & \multirow{2}{*}{$\begin{array}{c}\text { Mean difference } \\
\text { IV, random, 95\% CI }\end{array}$} & \multirow{2}{*}{\multicolumn{4}{|c|}{$\begin{array}{c}\text { Mean difference } \\
\text { IV, random, 95\% CI }\end{array}$}} \\
\hline & Mean & SD & Total & Mean & $\mathrm{SD}$ & Total & & & & & & \\
\hline & 0.73 & 0.096 & 27 & 0.68 & 0.074 & 30 & 20.3 & $0.05[0.01,0.09]$ & & & $\longrightarrow$ & \\
\hline G. Dischereit, et al. 2016 & 0.84 & 0.1 & 25 & 0.81 & 0.12 & 17 & 17.8 & $0.03[-0.04,0.10]$ & & & & \\
\hline Li R, et al. 2019 & 0.803 & 0.045 & 26 & 0.755 & 0.057 & 26 & 21.7 & $0.05[0.02,0.08]$ & & & - & \\
\hline Li XQ, et al. 2016 & 0.83 & 0.14 & 94 & 0.64 & 0.15 & 94 & 20.6 & $0.19[0.15,0.23]$ & & & & - \\
\hline Xu ML, 2017 & 0.8 & 0.15 & 50 & 0.65 & 0.12 & 50 & 19.5 & $0.15[0.10,0.20]$ & & & & - \\
\hline \multicolumn{3}{|l|}{ Total (95\% CI) } & 222 & & & 217 & 100.0 & $0.09[0.03,0.16]$ & & & & \\
\hline \multicolumn{9}{|c|}{ Heterogeneity: $\operatorname{tau}^{2}=0.00 ; \mathrm{chi}^{2}=41.78, d f=4(P<0.00001) ; I^{2}=90 \%$} & $T$ & $T$ & 1 & $T$ \\
\hline \multirow{2}{*}{\multicolumn{9}{|c|}{ Test for overall effect: $Z=2.90(P=0.004)$}} & -0.2 & -0.1 & 0.1 & 0.2 \\
\hline & & & & & & & & & \multicolumn{4}{|c|}{ Favours [control] $\quad$ Favours [kinesitherapy } \\
\hline
\end{tabular}

FIGURE 6: Meta-analysis of femoral neck BMD.

involved $21 \mathrm{RCTs}$ that included a total of 1840 subjects with POP (including SOP and PMOP). The duration of treatment varied from 3 to 24 months. The outcome measures primarily consisted of lumbar spine and femoral neck BMD. The results of the meta-analysis showed that there were no statistically significant differences between kinesitherapy plus antiosteoporosis medications versus antiosteoporosis medications alone on lumbar spine BMD when the duration of intervention was less than 6 months $(\mathrm{MD}=0.02$; 95\% CI: $-0.00-0.05 ; P=0.10)$. However, kinesitherapy had a positive effect on lumbar spine BMD when the duration of intervention was 6 months $\left(\mathrm{MD}=0.11 \mathrm{~g} / \mathrm{cm}^{2} ; 95 \% \mathrm{CI}\right.$ : $0.06-0.16$; $P<0.0001)$ or more than 6 months $\left(\mathrm{MD}=0.04 \mathrm{~g} / \mathrm{cm}^{2} ; 95 \% \mathrm{CI}\right.$ : $0.02-0.06 ; P<0.0001)$ compared with antiosteoporosis medications alone. Furthermore, kinesitherapy had a remarkable effect on femoral neck BMD $\left(\mathrm{MD}=0.09 \mathrm{~g} / \mathrm{cm}^{2} ; 95 \% \mathrm{CI}\right.$ : $0.03-0.16 ; P<0.004)$ when compared with antiosteoporosis medications alone.

4.2. Limitations and Suggestions for Future Research. A total of 21 RCTs were included in this review, which showed that kinesitherapy had a favourable effect on lumbar spine and femoral neck BMD in patients with POP. Nevertheless, the interpretation and generalization of this systematic review and meta-analysis are subject to some limitations. According to the Cochrane Collaboration's tool, low-quality evidence, which included studies with a high risk of bias, resulted in a high heterogeneity of the meta-analysis results and favoured the positive effect of kinesitherapy on BMD in patients with POP. There were eleven RCTs that did not report the random sequence generation, and the remaining RCTs were lacking detailed descriptions of randomization, which could result in selection bias. The performance bias was high since the blinding of participants and personnel was not implemented. In all of the included trials, whether the blinding of the outcome assessment was used or not is not mentioned except for one study which states explicitly that the blinding of the outcome assessment was not applied and the statistics of outcome operated by the author. Although one trial reported reasons for withdrawal and dropout, an intentionto-treat analysis was not performed in the data analysis phase for which attrition bias was inevitable. In addition, all of the study protocols from the trials could not be obtained. Furthermore, a specific exercise was not designed for the analysis, which suggests that such an analysis is problematic due to the diversity of interventions. Only five trials included the outcome of femoral neck BMD in the currently available 
studies; thus, the reliability of the treatment effects of kinesitherapy on femoral neck BMD is reduced. Therefore, more multicenter, larger sample, long-term, single-blind RCTs are required to assess the effect of kinesitherapy on $\mathrm{BMD}$ in patients with POP.

\section{Conclusion}

The meta-analyses in this study suggest that kinesitherapy plus antiosteoporosis medications can significantly improve lumbar spine BMD when the duration of intervention is longer than 6 months compared with antiosteoporosis medications alone in the current low-quality evidence. More high-quality evidence in the form of multicenter, larger sample, long-term, single-blind, randomized controlled trials is required to confirm the effect of kinesitherapy on $\mathrm{BMD}$ in patients with POP.

\section{Conflicts of Interest}

The authors have no conflicts of interest to declare.

\section{Acknowledgments}

This study was supported by the Youth Program of the Department of Education, Hunan Province, China (grant no. 17B246).

\section{References}

[1] Y. Jiang, Y. Pei, X. Y. Miao et al., "Relationship among aging,body composition,body mass index, and bone mass density in elderly men over 50-year-old," Chinese Journal of Osteoporosis \& Bone Mineral Research, vol. 9, no. 2, pp. 122-128, 2016.

[2] K. S. Park, J. I. Yoo, H. Y. Kim et al., "Education and exercise program improves osteoporosis knowledge and changes calcium and vitamin D dietary intake in community dwelling elderly," Bmc Public Health, vol. 17, no. 1, p. 966, 2017.

[3] M. R. Mcclung, "Prevention and management of osteoporosis," Best Practice \& Research Clinical Endocrinology \& Metabolism, vol. 17, no. 1, pp. 53-71, 2003.

[4] H. J. Choi, C. S. Shin, Y.-C. Ha et al., "Burden of osteoporosis in adults in Korea: a national health insurance database study," Journal of Bone and Mineral Metabolism, vol. 30, no. 1, pp. 54-58, 2012.

[5] C. García-Gomáriz, J. M. Blasco, C. Macián-Romero, E. Guillem-Hernández, and C. Igual-Camacho, "Effect of 2 years of endurance and high-impact training on preventing osteoporosis in postmenopausal women," Menopause, vol. 25, no. 3, pp. 301-306, 2018.

[6] L. Sung-Rak, H. Yong-Chan, H. Kang et al., "Morbidity and mortality in jeju residents over 50-years of age with hip fracture with mean 6-year follow-up: a prospective cohort study," Journal of Korean Medical Science, vol. 28, no. 7, pp. 1089-1094, 2013.

[7] J. L. Porter and S. S. Bhimji, Osteoporosis. Treasure Island, StatPearls Publishing, Treasure Island, FL, USA, 2017.

[8] E. Gielen, D. Vanderschueren, F. Callewaert, and S. Boonen, "Osteoporosis in men," Best Practice \& Research Clinical Endocrinology \& Metabolism, vol. 25, no. 2, pp. 321-335, 2011.
[9] D. Bliuc, N. D. Nguyen, D. Alarkawi, T. V. Nguyen, J. A. Eisman, and J. R. Center, "Accelerated bone loss and increased post-fracture mortality in elderly women and men," Osteoporosis International, vol. 26, no. 4, pp. 1331-1339, 2015.

[10] N. C. Wright, A. C. Looker, K. G. Saag et al., "The recent prevalence of osteoporosis and low bone mass in the United States based on bone mineral density at the femoral neck or lumbar spine," Journal of Bone and Mineral Research, vol. 29, no. 11, pp. 2520-2526, 2014.

[11] W. D. Leslie, L. M. Lix, G. S. Finlayson, C. J. Metge, S. N. Morin, and S. R. Majumdar, "Direct healthcare costs for 5 years post-fracture in Canada," Osteoporosis International, vol. 24, no. 5, pp. 1697-1705, 2013.

[12] R. Liu and B. K. Zhao, "The drug treatment of Osteoporosis," Chinese Journal of Ethnomedicine \& Ethnopharmacy, vol. 4, no. 7, pp. 58-60, 2014.

[13] W. Wang, N. I. Li-Gang, L. I. Chun-Wen et al., "Research progress of osteoporotic hip fracture in old age," Chinese Archives of Traditional Chinese Medicine, vol. 30, no. 5, pp. 1069-1072, 2012.

[14] K. Sundeep and P. Roberto, Osteoporosis, Elsevier, Amsterdam, Netherlands, 4th edition, 2013.

[15] A. Svedbom, E. Hernlund, M. Ivergård et al., "Osteoporosis in the European Union: a compendium of country-specific reports," Archives of Osteoporosis, vol. 8, no. 1-2, p. 136, 2013.

[16] S. Maria and P. A. Witt-Enderby, "Melatonin effects on bone: potential use for the prevention and treatment for osteopenia, osteoporosis, and periodontal disease and for use in bonegrafting procedures," Journal of Pineal Research, vol. 56, no. 2, pp. 115-125, 2014.

[17] Z. Porhashem, M. Biani, H. Noreddini et al., "Prevalence of osteoporosis and its association with serum vitamin D level in older people in Amirkola, North of Iran," Caspian Journal of Internal Medicine, vol. 3, no. 1, pp. 347-353, 2012.

[18] Z. M. Hu and C. Liu, "Epidemiology and associated risk factors of osteoporosis," World Latest Medicine Information, vol. 19, no. 42, pp. 55-57, 2019.

[19] I. R. Reid, D. M. Black, R. Eastell et al., "Reduction in the risk of clinical fractures after a single dose of zoledronic acid 5 milligrams," Bone, vol. 48, no. 2, pp. 557-563, 2011.

[20] T. E. Howe, B. Shea, L. J. Dawson et al., "Exercise for preventing and treating osteoporosis in postmenopausal women," The Cochrane Database of System Reviews, vol. 6, no. 7, Article ID CD000333, 2011.

[21] J. Iwamoto, "A role of exercise and sports in the prevention of osteoporosis," Clinical Calcium, vol. 27, no. 1, pp. 17-23, 2017.

[22] R. Zhao, M. Zhang, and Q. Zhang, "The effectiveness of combined exercise interventions for preventing postmenopausal bone loss: a systematic review and meta-analysis," Journal of Orthopaedic \& Sports Physical Therapy, vol. 47, no. 4, pp. 241-251, 2017.

[23] L. Cheng, S. Yan, J. Zhu, P. Cai, T. Wang, and Z. Shi, "Exercise enhance the ectopic bone formation of calcium phosphate biomaterials in muscles of mice," Materials Science and Engineering: C, vol. 77, pp. 136-141, 2017.

[24] W. A. van Gemert, A. J. Schuit, J. van der Palen et al., "Effect of weight loss, with or without exercise, on body composition and sex hormones in postmenopausal women: the shape-2 trial," Breast Cancer Research, vol. 17, p. 120, 2015.

[25] M. de Roon, A. M. May, A. McTiernan et al., "Effect of exercise and/or reduced calorie dietary interventions on breast cancerrelated endogenous sex hormones in healthy postmenopausal women," Breast Cancer Research, vol. 20, no. 1, p. 81, 2018. 
[26] R. Armamento-Villareal, L. Aguirre, D. L. Waters et al., "Effect of aerobic or resistance exercise, or both, on bone mineral density and bone metabolism in obese older adults while dieting: a randomized controlled trial," Journal of Bone and Mineral Research, vol. 35, no. 3, pp. 430-439, 2019.

[27] C. F. Dionello, D. Sá-Caputo, H. V. Pereira et al., "Effects of whole body vibration exercises on bone mineral density of women with postmenopausal osteoporosis without medications: novel findings and literature review," Journal of Musculoskeletal \& Neuronal Interactions, vol. 16, no. 3, pp. 193-203, 2016.

[28] R. Zhao, Z. Xu, and M. Zhao, "Antiresorptive agents increase the effects of exercise on preventing postmenopausal bone loss in women: a meta-analysis," Plos One, vol. 10, no. 1, Article ID e0116729, 2015.

[29] D. Segev, D. Hellerstein, and A. Dunsky, "Physical activitydoes it really increase bone density in postmenopausal women? A review of articles published between 2001-2016," Current Aging Science, vol. 11, no. 1, pp. 4-9, 2018.

[30] A. Varahra, I. B. Rodrigues, J. C. Macdermid et al., "Exercise to improve functional outcomes in persons with osteoporosis: a systematic review and meta-analysis," Osteoporosis International, vol. 29, no. 1, pp. 1-22, 2018.

[31] J. Savović, L. Weeks, J. A Sterne et al., "Evaluation of the cochrane collaboration's tool for assessing the risk of bias in randomized trials: focus groups, online survey, proposed recommendations and their implementation," Systematic Reviews, vol. 3, p. 37, 2014.

[32] G. Zheng, S. Li, M. Huang et al., "The effect of tai chi Training on cardiorespiratory fitness in healthy adults: a systematic review and meta-analysis," Plos One, vol. 10, no. 2, Article ID e0117360, 2015.

[33] J. P. T. Higgins, S. G. Thompson, J. J. Deeks et al., "Measuring inconsistency in meta-analyses," Bmj, vol. 327, no. 7414, pp. 557-560, 2003.

[34] J. Iwamoto, T. Takeda, and S. Ichimura, "Effect of exercise training and detraining on bone mineral density in postmenopausal women with osteoporosis," Journal of Orthopaedic Science, vol. 6, no. 2, pp. 128-132, 2001.

[35] C. F. Shen, "Clinical study on the treatment of primary osteoporosis (POP) by combined method of move and nourish the kidney," Hunan College of Traditional Chinese Medicine, 2003.

[36] H. L. Zhu, "Effects of exercise on bone mass and bone metabolism in elderly patients with osteoporosis," Maternal and Child Health Care of China, vol. 9, pp. 1251-1252, 2007.

[37] H. Q. Liu, J. J. Qin, and H. J. Liu, "Effect of combined kinesitherapy on bone mineral density in postmenopausal osteoporosis," The Journal of Traditional Chinese Orthopedics and Traumatology, vol. 19, no. 3, p. 14, 2007.

[38] H. B. Li, Q. Mei, X. L. Yin, and R. Chang, "Clinical observation of exercise and bone peptide preparation on bone mineral density in patients of osteoporosis," Journal of Henan Medical College, vol. 20, no. 5, pp. 446-447, 2008.

[39] X. X. Liu, Y. Zhou, and Y. P. Han, "Effects of community intervention on bone density in elderly patients with osteoporosis," Guangzhou Medical Journal, vol. 40, no. 3, pp. 67-68, 2009.

[40] L. L. Liu and J. Wang, "Observation of the efficacy of oral supplying compound calcium and exercise prescription for the treatment of senile osteoporosis," Journal of Clinical Research, vol. 29, no. 4, pp. 743-745, 2012.

[41] C. X. Li, Y. J. Tang, F. Y. Huang et al., "The effect of progressive psoas muscle functional exercise on bone density and low back pain in patients with osteoporosis," Chinese Journal of Gerontology, vol. 33, no. 15, pp. 3623-3624, 2013.

[42] Q. X. Ming, "Analysis on the effect of exercise rehabilitation on senile osteoporosis," China Health Care \& Nutrition, vol. 23, no. 10, pp. 5735-5736, 2013.

[43] H. Chen, Exercise Therapy Has the Influence of Bone Mineral Density and Biochemical Index for Patients of Postmenopausal with Osteoporosis, Nanjing University of Chinese Medicine, Nanjing, China, 2015.

[44] G. Dischereit, U. Müller-Ladner, and U. Lange, "Effects of osteoporosis specific standardized physical therapy on functional capacity, bone mineral density and bone metabolism-a 2-year prospective and randomized study," Physikalische Medizin, Rehabilitationsmedizin, Kurortmedizin, vol. 26, no. 3, pp. 124-129, 2016.

[45] X. Q. Li, L. N. Tao, and H. Y. Ren, "Effect of estrogen replacement therapy combined with kinesitherapy on postmenopausal osteoporosis in women," Laboratory Medicine and Clinic, vol. 13, no. 21, pp. 3099-3101, 2016.

[46] C. Chen, Effect of Exercise Therapy on Bone Mineral Density, lean Body Mass and Fat Mass of Postmenopausal Osteoporosis, Nanjing University of Chinese Medicine, Nanjing, China, 2016.

[47] M. L. Xu, "Effect of hormone replacement combined with exercise therapy on osteoporosis in postmenopausal women," Chinese And Foreign Medical Research, vol. 15, no. 22, pp. 50-51, 2017.

[48] L. Chang, "Effect of alternating exercise therapy on the rehabilitation of senile osteoporosis patients," Chinese Journal of Geriatric Care, vol. 15, no. 5, pp. 79-80, 2017.

[49] X. Qi, "The preventive effect of exercise therapy on osteoporosis fracture in postmenopausal women," China Reflexolocy, vol. 26, no. 10, pp. 158-159, 2017.

[50] Z. Wen, "The role of exercise therapy in the prevention of osteoporosis fracture in elderly women," World Latest Medicine Information, vol. 17, no. 39, p. 156, 2017.

[51] M. Liu and J. Q. Yang, "Effect of long-term aerobic exercise on estrogen level and bone composition in female patients with osteoporosis," Journal of Hainan Medical University, vol. 24, no. 18 , pp. 71-77, 2018.

[52] L. Q. Zheng, X. A. N. Pan, and Y. Q. Zheng, "Effect of alternating exercise therapy on the rehabilitation of senile osteoporosis patients," Jilin Medical Journal, vol. 40, no. 6, pp. 1320-1321, 2019.

[53] R. Li, Z. Yang, W. Han et al., "Effects of combined exercise therapy on bone metabolism in postmenopausal patients with osteoporosis," International Journal of Orthopaedics, vol. 40, no. 1, pp. 56-66, 2019.

[54] Y. Yan and F. Zhao, "Effect of exercise intervention on clinical effect, back pain and bone metabolism of osteoporosis patients," Investigacion Clinica, vol. 60, no. 5, pp. 1219-1231, 2019.

[55] C. L. Lai, S. Y. Tseng, C. N. Chen et al., "Effect of 6 months of whole body vibration on lumbar spine bone density in postmenopausal women: a randomized controlled trial," Clinical Interventions in Aging, vol. 8, pp. 1603-1609, 2013.

[56] S. S. Maeda and M. Lazaretti-Castro, "An overview on the treatment of postmenopausal osteoporosis," Arquivos Brasileiros de Endocrinologia \& Metabologia, vol. 58, no. 2, pp. 162-171, 2014.

[57] L. M. Giangregorio, A. Papaioannou, N. J. Macintyre et al., "Too fit to fracture: exercise recommendations for individuals with osteoporosis or osteoporotic vertebral fracture," Osteoporosis International, vol. 25, no. 3, pp. 821-835, 2014. 
[58] Al-S. A. ShanbE. F. Youssef et al., "The effect of magnetic therapy and active exercise on bone mineral density in elderly women with osteoporosis," Journal of Musculoskeletal Research, vol. 15, no. 3, pp. 65-70, 2012.

[59] E. Angin, Z. Erden, and F. Can, "The effects of clinical pilates exercises on bone mineral density (BMD), physical performance and quality of life of women with postmenopausal osteoporosis," Journal of Back \& Musculoskeletal Rehabilitation, vol. 28, no. 4, p. 849, 2015. 